\title{
AUTOMATIC SPEECHREADING WITH APPLICATION TO SPEAKER VERIFICATION
}

C. C. Broun

\begin{abstract}
Speech not only conveys the linguistic information, but also characterizes the talker's identity and therefore can be used in personal authentication. While most of the speech information is contained in the acoustic channel, the lip movement during speech production also provides useful information. In this paper we investigate the effectiveness of visual speech features in a speaker verifcation task. We frst present the visual front-end of the automatic speechreading system. We then develop a recognition engine to train and recognize sequences of visual parameters. The experimental results based on the XM2VTS database [1] demonstrate that visual information is highly effective in reducing both false acceptance and false rejection rates in speaker verifcation tasks.
\end{abstract}

\section{INTRODUCTION}

While speech primarily conveys the linguistic information, it also characterizes a talker's identity and, therefore, can be used for personal authentication. There is an increasing requirement for reliable personal authentication systems in areas of high security or secure access. The use of speech to identify a person has the advantage of requiring little custom hardware and is non-intrusive. However there are two signifcant problems in current generation speaker verifcation systems using speech. One is the diffculty in acquiring clean audio signals in an unconstrained environment. The other is that unimodal biometric models do not always work well with a signifcant percentage of the population. To combat these issues, systems incorporating the visual modality are being investigated to improve system robustness to environmental conditions, as well as to improve overall accuracy across the population. Face recognition has been an active research area during the past few years. However, face recognition is often based on static face images assuming a neutral facial expression and requires that the speaker not have signifcant appearance changes. Lip movement is a natural by-product of speech production, and it not only redects speaker dependent static and dynamic features, but also provides "liveness" testing.

\author{
X. Zhang, R. M. Mersereau, M. Clements
}

In this work, we incorporate visual speech features into a speaker verifcation system to assess the beneft of the visual modality. The paper is organized as follows. Section 2 presents our visual front end for the automatic speechreading system. The problem of speaker recognition using bimodal speech information is investigated in Section 3. Finally, Section 4 offers our conclusions.

\section{VISUAL ANALYSIS}

It is generally agreed that most visual speech information is contained in the lips. Thus, visual analysis focuses on lip feature extraction. The frst stage of the visual analysis involves a region of interest (ROI) detection - locating the speaker's mouth in a video sequence.

In another study [2], we use hue and saturation thresholding to locate the lips. Although it works well for most cases, complications might occur when distracting red blobs exist in the clothing, or if the person has a ruddy complexion. To eliminate these non-lip red blobs, we developed strategies using gradient/geometric/saturation constraints [3].

In the current paper we propose another solution that uses motion cues. Since a talking person's mouth is moving, we search for the moving lips in the image if an audio signal is present in the acoustic channel. To detect the moving object, we build difference images between subsequent frames and sum over a series of frames. The accumulated difference image (ADI) is defned as follows:

$$
\begin{aligned}
& A D I_{0}(x, y)=0 \\
& A D I_{k}(x, y)=A D I_{k-1}(x, y)+\Delta R_{k}(x, y), k \in 1, \cdots T
\end{aligned}
$$

where the difference image $\Delta R_{k}(x, y)$ is calculated by pixelwise absolute subtraction between two adjacent frames $\Delta R_{k}(x, y)=\left|R_{k}(x, y)-R_{k-1}(x, y)\right|$. Note we use the $\mathrm{R}$ component for our lip detection. $T$ is set to 30 in this work. An example of an accumulated image is shown in Fig. 1(d). To separate the moving lips from the background, we require all pixels exceeding a certain value be assigned to 1 , and 0 otherwise. We determine this threshold by using Otsu's method [4]. The resulting binary image is shown 


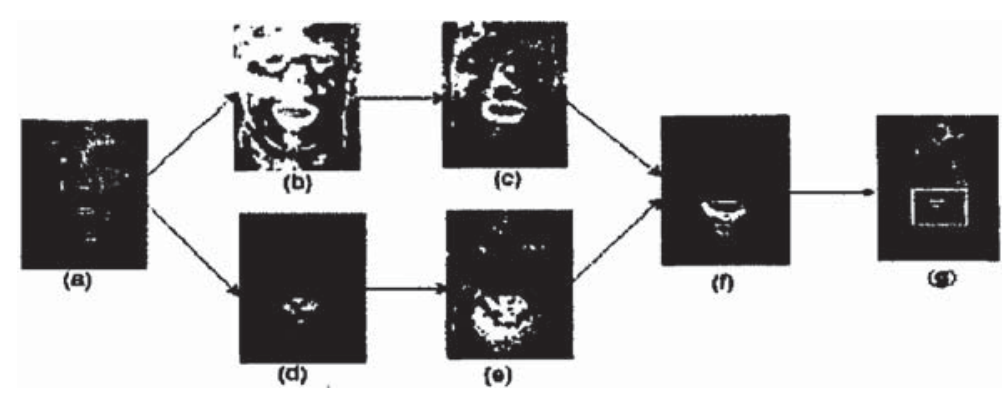

Fig. 1. Visual speech ROI detection (a): Gray level representation of original RGB color image. (b): Hue image. (c): Binary image after H/S thresholding. (d): Accumulated difference image. (e): Binary image after thresholding on (d). (f): Result from AND-operation on (c) and (e). (g): Original image with the identifed lip region.

in Fig. 1(e). Fig. 1(f) is obtained by using the AND operation to combine the binary image from the hue/saturation thresholding [2], as shown in Fig. 1(c), with the accumulated difference image. Based on the resulting image, we extract the lip region from its surroundings by fnding the largest connected region. The identifed lip area is shown as a white bounding box in Fig. 1(g). Subsequent processing is restricted to the identifed lip region.

To derive the lip dimensions within the ROI, we make use of both color and edge information of an image. We developed an algorithm which uses a Markov random feld framework to combine these two types of information and segment the lips from the background. Details of the MRFbased lip segmentation can be found in [5].

Fig. 2 shows examples of segmentation results with different persons and different lip opening situations. We observe that the highlighted pixels fairly well match the true lip area. Based on the segmented lip image, it is easy to extract the key feature points on the lips and subsequently derive the geometric dimensions of the lips. The following features are used in our study: mouth width $\left(w_{2}\right)$, upper/lower lip width $\left(h_{1}, h_{3}\right)$, lip opening height/width $\left(h_{2}, w_{1}\right)$, and the distance between horizontal lip line and upper lip $\left(h_{4}\right)$. An illustration of the geometry is shown in Fig. 3. Besides the geometric dimensions of the lip, we also detect the visibility of the tongue and teeth [5]. The indicator of the tongue/teeth is set to 1 if they are detected, and 0 otherwise.
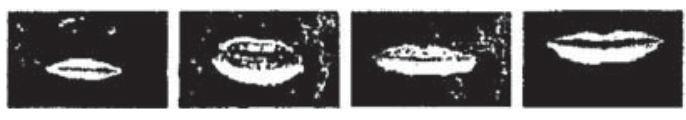

Fig. 2. Segmented lip overlayed on original image.

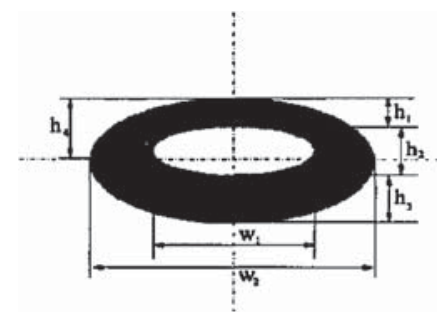

Fig. 3. Illustration of extracted geometric features of the lip.

\section{SPEAKER VERIFICATION}

Speaker verifcation seeks to validate a claimed identity, i.e., either to accept or reject an identity claim.

For the speaker verifcation task, we use the polynomialbased approach developed in [6]. Polynomial-based classifcation requires low computation while maintaining high accuracy. Because of the Weierstrass approximation theorem, polynomials are universal approximators for a Bayes classifer [7].

The classifer consists of several parts as shown in Fig. 4. The extracted features $x_{1}, \cdots, x_{N}$ are introduced to the classifer. For each feature vector $\mathbf{x}$, a score is produced by using the polynomial discriminant function $f(x)=w^{T} p(x)$, where $\mathbf{p}(\mathbf{x})$ is the polynomial basis vector constructed from the input vector $\mathbf{x}, \mathbf{p}(\mathbf{x})=\left[\begin{array}{llllll}1 & x_{1} & x_{2} & x_{1}^{2} & x_{1} x_{2} & x_{2}^{2}\end{array}\right]^{T}$ for a two-dimensional feature vector $\mathbf{x}=\left[\begin{array}{ll}x_{1} & x_{2}\end{array}\right]^{T}$ and for polynomial order two, and $w$ is the class model. The fnal score is computed by averaging over all feature vectors.

$$
\text { Score }=\frac{1}{N} \sum_{i=1}^{N} \mathrm{w}^{T} \mathrm{p}\left(\mathrm{x}_{i}\right) .
$$

The accept/reject decision is performed by comparing the output score to a threshold. If Score $<T$, then reject the 
claim, otherwise, accept the claim.

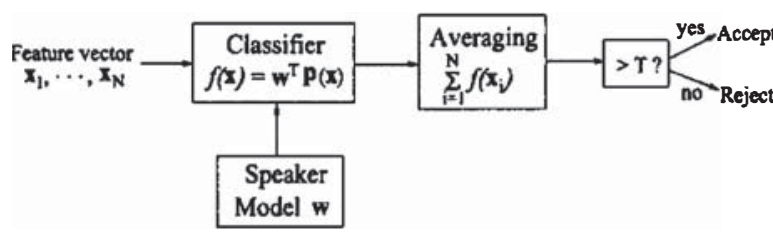

Fig. 4. Structure of a polynomial classifer.

We perform the speaker verifcation test on the XM2VTS database [1]. This database includes four recordings of 295 subjects taken at one month intervals. (However we were able to use only 261 of the 295 speakers because of corrupted audio or video sequences.) Each sequence is approximately 5 seconds long and contains the subject speaking the sentence "Joe took father's green shoe bench out." The database covers a large population from various ethnic origins and with various appearances. The same person might attend the four sessions with a different appearance, including hairstyles, with/without glasses, with/without beard, with/without lipstick.

To evaluate the performance of the person authentication systems on the XM2VTS database, we adopt the protocol defned in [8]. We chose confguration II due to the audio-visual data we are using. The protocol partitions the database into a training set, evaluation set and test set. In confguration II, data from the frst two sessions are used to train the clients' models. The system threshold is set from evaluation data composed of the third session of the clients' data and all four sessions of the evaluation imposters' data. The fnal performance test uses data from the fourth session of the clients and from all four sessions of the test imposters. For this description, each subject appears only in one set. This ensures realistic evaluation of the imposter claims whose identity is unknown to the system.

The verifcation performance is characterized by two error rates computed during tests: the false acceptance rate (FAR) and the false rejection rate (FRR). The pooled equal error rate (EER) threshold is determined from the evaluation set and used against the test population to determine the system performance. Both FAR and FRR are reported for this EER operating point. The test results for a visual only speaker verifcation system are shown below:

\begin{tabular}{|c|c|c|c|}
\hline Features & Poly. Order & FRR \% & FAR \% \\
\hline all (8) & 2 & 8.8 & 9.7 \\
\hline all $+\Delta(16)$ & 2 & 6.1 & 9.3 \\
\hline all (8) & 3 & 5.0 & 9.0 \\
\hline all+ $\Delta(16)$ & 3 & 4.4 & 8.2 \\
\hline all+time (9) & 2 & 8.3 & 9.2 \\
\hline all+time (9) & 3 & 4.8 & 8.5 \\
\hline
\end{tabular}

In our experiment, polynomial orders two and three are used. The visual features included are the eight parameters derived in the last section. Extra features are the corresponding delta features and the normalized time index $i / M$, where $i$ is the current frame index, and $M$ is the total number of frames. As can be seen, by incorporating extra features, a lower error rate is achieved. At the same time, increasing the polynomial order also contributes to improved verifcation results.

In the audio modality, each feature vector is composed of 12 cepstral coefEcients and one normalized time index [3]. A third-order polynomial classifer is used. To fuse the two modalities, we use late integration. It is necessary that the classifer outputs represent class probabilities. We use an optimum Bayes approach. First we calculate the conditional probability $p\left(\mathbf{x}_{1}, \cdots, \mathbf{x}_{N} \mid \omega_{j}\right)$, which we abbreviate as $p\left(\mathbf{x}_{1}^{N} \mid \omega_{j}\right)$, where $\omega_{j}$ is class $j$. By assuming independence, we obtain

$$
p\left(\mathbf{x}_{1}^{N} \mid \omega_{j}\right)=\prod_{k=1}^{N} p\left(\mathbf{x}_{k} \mid \omega_{j}\right) .
$$

Using the relation

$$
p\left(\mathbf{x}_{k} \mid \omega_{j}\right)=\frac{p\left(\omega_{j} \mid \mathbf{x}_{k}\right) p\left(\mathbf{x}_{k}\right)}{p\left(\omega_{j}\right)}
$$

and (2), we obtain the discriminant function

$$
d^{\prime}\left(\mathbf{x}_{1}^{N}\right)=\prod_{k=1}^{N} \frac{p\left(\omega_{j} \mid \mathbf{x}_{k}\right)}{p\left(\omega_{j}\right)} .
$$

We have discarded the numerator term $\prod_{k=1}^{N} p\left(\mathbf{x}_{k}\right)$ because it is independent of $\omega_{j}$. Two simplifcations are performed. First we consider the logarithm of the discriminant function,

$$
\log \left(d^{\prime}\left(\mathbf{x}_{1}^{N}\right)\right)=\sum_{k=1}^{N} \log \frac{p\left(\omega_{j} \mid \mathbf{x}_{k}\right)}{p\left(\omega_{j}\right)} .
$$

Using a Taylor series, a linear approximation of $\log (x)$ around $x=1$ is $x-1$. Thus, we can approximate $\log \left(d^{\prime}(x)\right)$ as

$$
d\left(\mathbf{x}_{1}^{N}\right)=\sum_{k=1}^{N} \frac{p\left(\omega_{j} \mid \mathbf{x}_{k}\right)}{p\left(\omega_{j}\right)}
$$

where we have dropped the -1 , since a constant offset will be eliminated in a log likelihood ratio function. Thus, the scoring method is equivalent to computing a log probability. We can combine the classifer output from the audio and visual modalities by averaging the class scores, $s=\alpha s_{A}+$ $(1-\alpha) s_{V}$. For the following experiments, the audio and visual modalities are weighted equally (i.e., $\alpha=0.5$ ). 


\section{FRR and FAR at EER in Varying Noise Conditions}

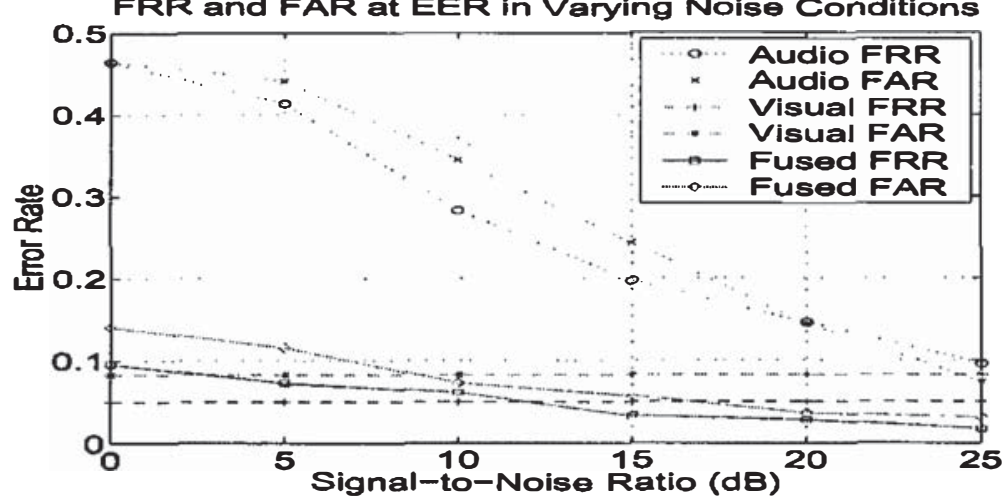

Fig. 5. Performance of audio-visual speaker verifcation in noisy conditions.

The performance of the bimodal speaker verifcation system is shown in Fig. 5. As can be seen, the performance of the audio modality degrades as the noise level increases. This fgure shows the False Rejection Rate (FRR) and the False Acceptance Rate (FAR) for each modality independently, as well as for the fused system. Both curves are of interest since the threshold is determined with an evaluation population separated from the test population. As illustrated in the fgure, the audio-visual fusion is shown to outperform both modalities at high signal-to-noise ratios.

\section{SUMMARY AND CONCLUSIONS}

In this paper we have demonstrated that visual speech features can be successfully used as an aid for personal authentication. Experiments based on the XM2VTS database [1] with 261 speakers achieve an FRR of $4.4 \%$ and an FAR of $8.2 \%$ with polynomial order three and suggest that visual information is highly effective for reducing the false acceptance and false rejection rates in such tasks. The combined audio-visual system is shown to outperform both modalities at high signal-to-noise ratios. However, error rates over the range of signal-to-noise ratios (SNR) indicate that a dynamic fusion strategy, e.g., adjusting the weighting of the modalities as SNR degrades, may improve the overall system performance.

\section{Acknowledgments}

The research on which this paper is based acknowledges the use of the Extended Multimodal Face Database and associated documents $[1,9]$.

\section{REFERENCES}

[1] "URL: ee.surrey.ac.uk/research/vssp/xm2vtsdb," .

[2] X. Zhang and R. M. Mersereau, "Lip feature extraction towards an automatic speechreading system," in Proc. IEEE ICIP, 2000.

[3] C. C. Broun and X. Zhang, "Multimodal fusion of polynomial classifers for automatic person recognition," in SPIE 15th AeroSense Symposium, April 2001.

[4] N. Otsu, "A threshold selection method from gray level histograms," IEEE Transactions on Systems, Man, and Cybernetics, vol. 9, pp. 62-66, 1979.

[5] X. Zhang, C. C. Broun, R. M. Mersereau, and M. Clements, "Automatic speechreading with applications to human-computer-interfaces," Submitted to EURASIP Joumal on Applied Signal Processing, Special issue on Audio-Visual Speech Processing, 2002.

[6] W. M. Campbell and C. C. Broun, "Low complexity speaker authentication techniques using polynomial classifers," in SPIE, 1999.

[7] J. Schuermann, Pattern Classifcation, John Wiley and Sons, Inc., 1996.

[8] J. Luettin and G. Maitre, "Evaluation protocol for the XM2VTS database," IDIAP-COM 98-05, October 1998.

[9] K. Messer, J. Matas, J. Kittler, J. Luettin, and G. Maitre, "XM2VTSDB: The Extended M2VTS Database," in Proceedings 2nd Conference on Audio and Video-Based Biometric Personal Verifcation (AVBPA99), 1999. 